\title{
207.
}

\section{ANALYTICAL SOLUTION OF THE PROBLEM OF TACTIONS.}

[From the Philosophical Magazine, vol. xıII. (1857), pp. 507-509.]

IT is well known that the eight circles, each of which touches three given circles, are determined as follows:-viz. considering any one in particular of the four axes of similitude of the given circles, and the perpendicular let fall from the radical centre (or centre of the orthotomic circle) of the given circles, there are two of the required tangent circles which have their centres upon the perpendicular, and pass through the points of intersection of the orthotomic circle and the axis of similitude, or in other words, the axis of similitude is a common chord (or radical axis) of the orthotomic circle and the two tangent circles. This suggests the choice of the radical centre for the origin of coordinates; and the resulting formulæ then take very simple forms, and the theorem is verified without difficulty.

Take then the centre of the orthotomic circle as the origin of coordinates, and let the radius of this circle be put equal to unity; then if $(\alpha, \beta),\left(\alpha^{\prime}, \beta^{\prime}\right),\left(\alpha^{\prime \prime}, \beta^{\prime \prime}\right)$ are the coordinates of the centres of the given circles, the equations of these will be

$$
\begin{aligned}
& x^{2}+y^{2}+1-2 \alpha x-2 \beta \quad y=0, \\
& x^{2}+y^{2}+1-2 \alpha^{\prime} x-2 \beta^{\prime} y=0, \\
& x^{2}+y^{2}+1-2 \alpha^{\prime \prime} x-2 \beta^{\prime \prime} y=0
\end{aligned}
$$

and the radii of the circles will be $\sqrt{\alpha^{2}+\beta^{2}-1}, \sqrt{\alpha^{\prime 2}+\beta^{\prime 2}-1}, \sqrt{\alpha^{\prime \prime 2}+\beta^{1 / 2}-1}$. It will be convenient to write

$$
\begin{aligned}
& \gamma= \pm \sqrt{\alpha^{2}+\beta^{2}-1} \\
& \gamma^{\prime}= \pm \sqrt{\alpha^{\prime 2}+\beta^{\prime 2}-1} \\
& \gamma^{\prime \prime}= \pm \sqrt{\alpha^{\prime \prime 2}+\beta^{\prime \prime 2}-1}
\end{aligned}
$$


where the three several signs \pm are fixed once for all in a determinate manner. If, however, all the signs are reversed, the result is the same, so that the system is one of four (not of eight) different systems. The coordinates of a centre of similitude of the second and third circles are

$$
\frac{\alpha^{\prime} \gamma^{\prime \prime}-\alpha^{\prime \prime} \gamma^{\prime}}{\gamma^{\prime \prime}-\gamma^{\prime}}, \frac{\beta^{\prime} \gamma^{\prime \prime}-\beta^{\prime \prime} \gamma^{\prime}}{\gamma^{\prime \prime}-\gamma^{\prime}}
$$

and forming the corresponding expressions for the coordinates of the centres of similitude of the third and first circles, and of the first and second eircles, the three centres of similitude lie on a line which will be an axis of similitude: to find the equation, write

$$
\begin{aligned}
& A=\beta \gamma^{\prime}-\beta^{\prime} \gamma+\beta^{\prime} \gamma^{\prime \prime}-\beta^{\prime \prime} \gamma^{\prime}+\beta^{\prime \prime} \gamma-\beta \gamma^{\prime \prime}, \\
& B=\gamma \alpha^{\prime}-\gamma^{\prime} \alpha+\gamma^{\prime} \alpha^{\prime \prime}-\gamma^{\prime \prime} \alpha^{\prime}+\gamma^{\prime \prime} \alpha-\gamma \alpha^{\prime \prime}, \\
& C=\alpha \beta^{\prime}-\alpha^{\prime} \beta+\alpha^{\prime} \beta^{\prime \prime}-\alpha^{\prime \prime} \beta^{\prime}+\alpha^{\prime \prime} \beta-\alpha \beta^{\prime \prime}, \\
& \nabla=\alpha \beta^{\prime} \gamma^{\prime \prime}-\alpha \beta^{\prime \prime} \gamma^{\prime}+\alpha^{\prime} \beta^{\prime \prime} \gamma-\alpha^{\prime} \beta \gamma^{\prime \prime}+\alpha^{\prime \prime} \beta \gamma^{\prime}-\alpha^{\prime \prime} \beta^{\prime} \gamma,
\end{aligned}
$$

values which, it will be observed, give

$$
\begin{aligned}
& A \alpha+B \beta+C \gamma=\nabla \\
& A \alpha^{\prime}+B \beta^{\prime}+C \gamma^{\prime}=\nabla, \\
& A \alpha^{\prime \prime}+B \beta^{\prime \prime}+C \gamma^{\prime \prime}=\nabla
\end{aligned}
$$

then the equation of the axis of similitude is found to be

$$
A x+B y-\nabla=0
$$

and hence the equation of the perpendicular let fall from the radical centre upon the axis of similitude is

$$
B x-A y=0 .
$$

It should therefore be possible to find two circles having their centres on the lastmentioned line and touching the three given circles Take $A \theta, B \theta$ as the coordinates of the centre of one of the two circles, and let $r$ be its radius; the conditions of tangency are

$$
\begin{aligned}
r & =\sqrt{(A \theta-\alpha)^{2}+(B \theta-\beta)^{2}} \pm \gamma, \\
& =\sqrt{\left(A \theta-\alpha^{\prime}\right)^{2}+\left(B \theta-\beta^{\prime}\right)^{2}} \pm \gamma^{\prime}, \\
& =\sqrt{\left(A \theta-\alpha^{\prime \prime}\right)^{2}+\left(B \theta-\beta^{\prime \prime}\right)^{2}} \pm \gamma^{\prime \prime},
\end{aligned}
$$

where the sign \pm has the same value in each expression. We have consequently

$$
(r \mp \gamma)^{2}=(A \theta-\alpha)^{2}+(B \theta-\beta)^{2} ;
$$

or, observing that $A \alpha+B \beta=\nabla-C \gamma$, and reducing,

$$
r^{2}-\left(A^{2}+B^{2}\right) \theta^{2}+2 \nabla \theta-1=2 \gamma( \pm r+C \theta) .
$$


Forming the two analogous equations, the three equations will be satisfied if only

$$
\begin{aligned}
r^{2}-\left(A^{2}+B^{2}\right) \theta^{2}+2 \nabla \theta-1 & =0 \\
\pm r+C \theta & =0 .
\end{aligned}
$$

Eliminating $r$, we have

$$
\left(A^{2}+B^{2}-C^{2}\right) \theta^{2}-2 \nabla \theta+1=0
$$

which gives for $\theta$ the two values

$$
\left(A^{2}+B^{2}-C^{2}\right) \theta=\nabla \pm \sqrt{\nabla^{2}-\left(A^{2}+B^{2}-C^{2}\right)} ;
$$

and then $r$ is determined linearly by the equation

$$
r=\mp C \theta \text {. }
$$

The equation of the tangent circle is therefore

$$
(x-A \theta)^{2}+(y-B \theta)^{2}=C^{2} \theta^{2} ;
$$

or reducing,

$$
x^{2}+y^{2}-1-2 \theta(A x+B y-\nabla)=0
$$

and recollecting that $A x+B y-\nabla=0$ is the equation of the axis of similitude, the equation shows that the axis of similitude is a common chord or radical axis of the orthotomic circle and the two tangent circles.

2, Stone Buildings, W.C., May 15, 1857. 